\title{
Article \\ Refinements of the Converse Hölder and Minkowski Inequalities
}

\author{
Josip Pečarić ${ }^{1}$, Jurica Perić ${ }^{2, *}$ and Sanja Varošanec ${ }^{3}$ \\ 1 Peoples Friendship University of Russia (RUDN University), 6 Miklukho-Maklaya Str., \\ 117198 Moscow, Russia; pecaric@element.hr \\ 2 Departments of Mathematics, Faculty of Science, University of Split, 21000 Split, Croatia \\ 3 Department of Mathematics, University of Zagreb, 10000 Zagreb, Croatia; varosans@math.hr \\ * Correspondence: jperic@pmfst.hr
}

check for updates

Citation: Pečarić, J.; Perić, J.; Varošanec, S. Refinements of the Converse Hölder and Minkowski Inequalities. Mathematics 2022, 10, 202. https://doi.org/10.3390/ math10020202

Academic Editor: Sotiris K. Ntouyas

Received: 11 December 2021 Accepted: 5 January 2022

Published: 10 January 2022

Publisher's Note: MDPI stays neutral with regard to jurisdictional claims in published maps and institutional affiliations.

Copyright: () 2022 by the authors. Licensee MDPI, Basel, Switzerland. This article is an open access article distributed under the terms and conditions of the Creative Commons Attribution (CC BY) license (https:// creativecommons.org/licenses/by/ $4.0 /)$.

\begin{abstract}
We give a refinement of the converse Hölder inequality for functionals using an interpolation result for Jensen's inequality. Additionally, we obtain similar improvements of the converse of the Beckenbach inequality. We consider the converse Minkowski inequality for functionals and of its continuous form and give refinements of it. Application on integral mixed means is given.
\end{abstract}

Keywords: Beckenbach's inequality; converse inequality; Hölder's inequality; mean; Minkowski's inequality; mixed mean

\section{Introduction}

In the last few decades, the study of classical inequalities, such as the Jensen, the Hölder, the Minkowski and similar inequalities has experienced great expansion. Many extensions, generalizations, improvements, refinements, and applications of these inequalities have been proved to support different research ideas. The first appearance of these inequalities was in integral and discrete forms. However, over the years, the settings in which they were appeared have expanded. For example, classical inequalities have been studied in measure spaces, in Hilbert spaces, on time scale, in families of set-valued mappings, etc. (for instance, see [1-9] and references given there). The connection point between numerous generalizations is the theory of isotonic linear functionals. Let us describe this term in detail.

Let $E$ be a non-empty set and $L$ be a linear class of real-valued functions $f$ on $E$ having the properties:

L1: $f, g \in L \Rightarrow(\alpha f+\beta g) \in L$ for all $\alpha, \beta \in \mathbb{R}$;

L2: $1 \in L$, i.e., if $f(t)=1$ for $t \in E$, then $f \in L$.

In this paper we consider a linear functional $A: L \rightarrow \mathbb{R}$ which is also isotonic, i.e., if $f \in L$, and $f \geq 0$ on $E$ then $A(f) \geq 0$. The basic examples of isotonic linear functionals are sum and R-integral.

Most classical inequalities have a variant involving a linear isotonic functional (see [9]). Among others, in [9] (p. 115) we can find the following converse Hölder inequality.

Theorem 1 (The converse Hölder inequality for functionals, [9]). Let L satisfy conditions L1 and $\mathrm{L} 2$ and let $A$ be an isotonic linear functional. Let $p>1, q=p /(p-1)$, and $w, f, g \geq 0$ on $E$ with $w f^{p}, w g^{q}, w f g \in L$. If $0<m \leq f(x) g^{-q / p}(x) \leq M$ for $x \in E$, then

$$
K(p, m, M) A^{\frac{1}{p}}\left(w f^{p}\right) A^{\frac{1}{q}}\left(w g^{q}\right) \leq A(w f g),
$$

where $K(p, m, M)$ is a constant defined as

$$
K(p, m, M)=|p|^{\frac{1}{p}}|q|^{\frac{1}{q}} \frac{(M-m)^{\frac{1}{p}}\left|m M^{p}-M m^{p}\right|^{\frac{1}{q}}}{\left|M^{p}-m^{p}\right|} .
$$


If $p<0$ or $0<p<1$, then the reverse inequality in (1) holds provided either $A\left(w f^{p}\right)>0$ or $A\left(w g^{q}\right)>0$.

Inequality (1) together with the Hölder inequality gives the following chain of inequalities

$$
K(p, m, M) A^{\frac{1}{p}}\left(w f^{p}\right) A^{\frac{1}{q}}\left(w g^{q}\right) \leq A(w f g) \leq A^{\frac{1}{p}}\left(w f^{p}\right) A^{\frac{1}{q}}\left(w g^{q}\right)
$$

in which we obtain the lower and the upper bound of the middle term $A(w f g)$. It is interesting to find more tighter estimation, i.e., to find refinements of the above inequality. In this article, we will focus on finding the refinement of the left side of inequality (3).

Let us say few words about organization of the paper. In Section 2 we give a refinement of the converse Hölder inequality using interpolation result from [10] (p. 717). Section 3 is devoted to the Beckenbach inequality. Finally, we consider the Minkowski inequality for infinitely many functions and for functionals, state its converse, give refinements of both variants of the converse Minkowski inequality and applied on integral mixed means.

\section{Refinement of the Converse Hölder Inequality}

The starting point of this consideration is the following interpolating inequality given in [10] (p. 717). It is proved by using the discrete Jensen inequality and, in fact, this result can be considered as a monotonicity property of the Jensen functional.

Theorem 2. If $\phi$ is a convex function on an interval $I \subseteq \mathbb{R}, x=\left(x_{1}, \ldots, x_{n}\right) \in I^{n}(n \geq 2)$, $p$ and $q$ are positive $n$-tuples such that $p_{i} \geq q_{i}$ for all $i=1,2, \ldots, n, P_{n}=\sum_{i=1}^{n} p_{i}, Q_{n}=\sum_{i=1}^{n} q_{i}$, then

$$
\begin{aligned}
\sum_{i=1}^{n} p_{i} \phi\left(x_{i}\right) & -P_{n} \phi\left(\frac{1}{P_{n}} \sum_{i=1}^{n} p_{i} x_{i}\right) \\
& \geq \sum_{i=1}^{n} q_{i} \phi\left(x_{i}\right)-Q_{n} \phi\left(\frac{1}{Q_{n}} \sum_{i=1}^{n} q_{i} x_{i}\right) \geq 0 .
\end{aligned}
$$

Additionally, let us recall the AG inequality in the following form:

Proposition 1 (The AG inequality). Let $a, b$ be positive real numbers. If $\alpha, \beta$ are positive real numbers, such that $\alpha+\beta=1$, then

$$
\alpha a+\beta b \geq a^{\alpha} b^{\beta}
$$

If $\alpha<0$ or $\alpha>1$, then the reversed inequality in (5) holds.

The main result of this paper is the following theorem which is a refinement of the known converse Hölder inequality (1). As we will see, its proof is based on the use of a new refinement of the AG inequality. This result has a key role in this paper.

Theorem 3. Let $A$ be a linear isotonic functional on a linear class L. Let $p \in \mathbb{R} \backslash\{1\}, q=\frac{p}{p-1}$, and $w, f, g \geq 0$ on $E$ with $w f^{p}, w g^{q}, w f g \in L$

Let $m, M$ be, such that $0<m \leq f(x) g^{-q / p}(x) \leq M$ for $x \in E$.

If $p>1$, then

$$
\begin{aligned}
A(w f g) & \geq K(p, m, M) A^{\frac{1}{p}}\left(w f^{p}\right) A^{\frac{1}{q}}\left(w g^{q}\right)+\Delta\left(g^{q}, f g\right) N(p, m, M) \\
& \geq K(p, m, M) A^{\frac{1}{p}}\left(w f^{p}\right) A^{\frac{1}{q}}\left(w g^{q}\right),
\end{aligned}
$$

where

$$
K(p, m, M)=|p|^{\frac{1}{p}}|q|^{\frac{1}{q}} \frac{(M-m)^{\frac{1}{p}}\left|m M^{p}-M m^{p}\right|^{\frac{1}{q}}}{\left|M^{p}-m^{p}\right|}
$$




$$
N(p, m, M)=\frac{m^{p}+M^{p}-2\left(\frac{m+M}{2}\right)^{p}}{M^{p}-m^{p}}
$$

and

$$
\Delta\left(g^{q}, f g\right)=A\left(w\left(\frac{M-m}{2} g^{q}-\left|f g-\frac{m+M}{2} g^{q}\right|\right)\right) .
$$

If $0<p<1$ and $A\left(w g^{q}\right)>0$, or $p<0$ and $A\left(w f^{p}\right)>0$, then the reversed inequalities in (6) and (7) hold.

Proof. Applying (4) with $p_{1}=\alpha, p_{2}=\beta$, where $\alpha$ and $\beta$ are positive real numbers, such that $\alpha+\beta=1, q_{1}=q_{2}=\min \{\alpha, \beta\}, \phi(x)=x^{p}, p>1$, we obtain the following inequality:

$$
(\alpha x+\beta y)^{p} \leq \alpha x^{p}+\beta y^{p}-\min \{\alpha, \beta\}\left(x^{p}+y^{p}-2\left(\frac{x+y}{2}\right)^{p}\right) .
$$

Let $h$ be a function from $L$, such that $0<m \leq h(x) \leq M$ for $x \in E, m \neq M$, and $\alpha$ and $\beta$ defined as

$$
\alpha(x)=\frac{M-h(x)}{M-m}, \quad \beta(x)=\frac{h(x)-m}{M-m} .
$$

Obviously, $\alpha(x)+\beta(x)=1, h(x)=\alpha(x) m+\beta(x) M$. Applying (8) with $x=m, y=M$, and the above-defined $\alpha(x)$ and $\beta(x)$ we obtain

$$
\begin{aligned}
h^{p}(x) & \leq \frac{M-h(x)}{M-m} m^{p}+\frac{h(x)-m}{M-m} M^{p} \\
& -\min \{\alpha(x), \beta(x)\}\left(m^{p}+M^{p}-2\left(\frac{m+M}{2}\right)^{p}\right) .
\end{aligned}
$$

Multiplying that inequality with $k(x) \geq 0$ and using linear functional $A$ it follows

$$
\begin{aligned}
A\left(k h^{p}\right) & \leq \frac{m^{p}}{M-m}(M A(k)-A(k h))+\frac{M^{p}}{M-m}(A(k h)-m A(k)) \\
& -A(k \min \{\alpha, \beta\})\left(m^{p}+M^{p}-2\left(\frac{m+M}{2}\right)^{p}\right) .
\end{aligned}
$$

Using formula $\min \{\alpha, \beta\}=\frac{1}{2}(\alpha+\beta-|\beta-\alpha|)$, replacing $h$ with $f g^{-\frac{q}{p}}$ and $k$ with $w g^{q}$, where $\frac{1}{p}+\frac{1}{q}=1$, after multiplying with $M-m$ we obtain

$$
\begin{aligned}
& (M-m) A\left(w f^{p}\right)+\left(m M^{p}-M m^{p}\right) A\left(w g^{q}\right) \\
+ & A\left(w\left(\frac{M-m}{2} g^{q}-\left|f g-\frac{m+M}{2} g^{q}\right|\right)\right)\left[m^{p}+M^{p}-2\left(\frac{m+M}{2}\right)^{p}\right] \\
\leq & \left(M^{p}-m^{p}\right) A(w f g) .
\end{aligned}
$$

In the following, the term $A\left(w\left(\frac{M-m}{2} F-\left|G-\frac{m+M}{2} F\right|\right)\right)$ is denoted by $\Delta(F, G)$.

Applying the AG inequality (5) with $\alpha=\frac{1}{p}>0, \beta=\frac{1}{q}>0, a=p(M-m) A\left(w f^{p}\right) \geq 0$ and $b=q\left(m M^{p}-M m^{p}\right) A\left(w g^{q}\right) \geq 0$ we have

$$
\begin{aligned}
& (M-m) A\left(w f^{p}\right)+\left(m M^{p}-M m^{p}\right) A\left(w g^{q}\right) \\
& =\frac{p}{p}(M-m) A\left(w f^{p}\right)+\frac{q}{q}\left(m M^{p}-M m^{p}\right) A\left(w g^{q}\right) \\
& \geq p^{\frac{1}{p}} q^{\frac{1}{q}}(M-m)^{\frac{1}{p}}\left(m M^{p}-M m^{p}\right)^{\frac{1}{q}} A^{\frac{1}{p}}\left(w f^{p}\right) A^{\frac{1}{q}}\left(w g^{q}\right) .
\end{aligned}
$$


Combining (9) and (10) and rearranging, it follows

$$
\begin{aligned}
& p^{\frac{1}{p}} q^{\frac{1}{q}}(M-m)^{\frac{1}{p}}\left(m M^{p}-M m^{p}\right)^{\frac{1}{q}} A^{\frac{1}{p}}\left(w f^{p}\right) A^{\frac{1}{q}}\left(w g^{q}\right) \\
& +\Delta\left(g^{q}, f g\right)\left[m^{p}+M^{p}-2\left(\frac{m+M}{2}\right)^{p}\right] \leq\left(M^{p}-m^{p}\right) A(w f g) .
\end{aligned}
$$

If $p>1$, then $M^{p}-m^{p}>0$, and after dividing with $M^{p}-m^{p}$ we obtain

$$
K(p, m, M) A^{\frac{1}{p}}\left(w f^{p}\right) A^{\frac{1}{q}}\left(w g^{q}\right)+\Delta\left(g^{q}, f g\right) N(p, m, M) \leq A(w f g),
$$

where $K(p, m, M)$ is a constant from (2) and $N(p, m, M)$ is a constant defined as

$$
N(p, m, M)=\frac{m^{p}+M^{p}-2\left(\frac{m+M}{2}\right)^{p}}{M^{p}-m^{p}} .
$$

Since the term $\Delta\left(g^{q}, f g\right) N(p, m, M)$ is non-negative for $p>1$ inequality (11) is an improvement of the converse Hölder inequality (1).

Let us discuss the other cases for the exponent $p$.

Let $p<0$. Then, the function $x \mapsto x^{p}$ is also convex on $\langle 0, \infty\rangle$, so inequality (9) holds. Additionally, we want to use the AG inequality, but now $\alpha<0, a<0$ and $b \leq 0$ since in this case $m M^{p}-M m^{p} \leq 0$. So, we have $\alpha a+\beta b=-(\alpha|a|+\beta|b|) \geq-|a|^{\alpha}|b|^{\beta}$ and

$$
\begin{aligned}
& (M-m) A\left(w f^{p}\right)+\left(m M^{p}-M m^{p}\right) A\left(w g^{q}\right) \\
& =-\left(\frac{1}{p}\left|p(M-m) A\left(w f^{p}\right)\right|+\frac{1}{q}\left|q\left(m M^{p}-M m^{p}\right) A\left(w g^{q}\right)\right|\right) \\
& \geq-|p|^{\frac{1}{p}}|q|^{\frac{1}{q}}(M-m)^{\frac{1}{p}}\left|m M^{p}-M m^{p}\right|^{\frac{1}{q}} A^{\frac{1}{p}}\left(w f^{p}\right) A^{\frac{1}{q}}\left(w g^{q}\right) .
\end{aligned}
$$

Using the above inequality together with (9) and multiplying with (-1) we obtain

$$
\begin{aligned}
& |p|^{\frac{1}{p}} q^{\frac{1}{q}}(M-m)^{\frac{1}{p}}\left|m M^{p}-M m^{p}\right|^{\frac{1}{q}} A^{\frac{1}{p}}\left(w f^{p}\right) A^{\frac{1}{q}}\left(w g^{q}\right) \\
& -\Delta\left(g^{q}, f g\right)\left[m^{p}+M^{p}-2\left(\frac{m+M}{2}\right)^{p}\right] \\
& \quad \geq \quad-\left(M^{p}-m^{p}\right) A(w f g)=\left|M^{p}-m^{p}\right| A(w f g) .
\end{aligned}
$$

A term $m^{p}+M^{p}-2\left(\frac{m+M}{2}\right)^{p}$ is positive because of the Jensen inequality for a strictly convex function $x \mapsto x^{p}, p<0$. After dividing with $\left|M^{p}-m^{p}\right|=-\left(M^{p}-m^{p}\right)$ it follows

$$
K(p, m, M) A^{\frac{1}{p}}\left(w f^{p}\right) A^{\frac{1}{q}}\left(w g^{q}\right)+\Delta\left(g^{q}, f g\right) N(p, m, M) \geq A(w f g) .
$$

In this case, the factor $N(p, m, M)$ is obviously negative.

Let $0<p<1$. Then, $x \mapsto x^{p}$ is concave on $[0, \infty)$ and in (9) reversed sign holds. Using the AG inequality with $\alpha=\frac{1}{p}>1, \beta=\frac{1}{q}<0, a=p(M-m) A\left(w f^{p}\right) \geq 0$ and $b=q\left(m M^{p}-M m^{p}\right) A\left(w g^{q}\right)=|q| \cdot\left|m M^{p}-M m^{p}\right| A\left(w g^{q}\right) \geq 0$ we obtain

$$
\begin{aligned}
& p^{\frac{1}{p}}|q|^{\frac{1}{q}}(M-m)^{\frac{1}{p}}\left|m M^{p}-M m^{p}\right|^{\frac{1}{q}} A^{\frac{1}{p}}\left(w f^{p}\right) A^{\frac{1}{q}}\left(w g^{q}\right) \\
& +\Delta\left(g^{q}, f g\right)\left[m^{p}+M^{p}-2\left(\frac{m+M}{2}\right)^{p}\right] \geq\left(M^{p}-m^{p}\right) A(w f g) .
\end{aligned}
$$

In this case, $M^{p}-m^{p}>0$ and dividing above inequality with $M^{p}-m^{p}$ inequality (13) follows.

Additionally, $m^{p}+M^{p}-2\left(\frac{m+M}{2}\right)^{p}$ is obviously negative, so the factor $N(p, m, M)$ is negative. 


\section{Refinement of the Converse Beckenbach Inequality}

One of the numerous generalizations of the Hölder inequality is the well-known Beckenbach inequality ([11]). Here we pay attention to the converse Beckenbach inequality. In [7] the following result (slightly modified) is given.

Theorem 4 (The converse Beckenbach inequality, [7]). Suppose that $\frac{1}{p}+\frac{1}{q}=1, a, b, c, x_{i}, y_{i}>0$ and $z_{i}=\left(\frac{a y_{i}}{b}\right)^{q / p},(i=1,2, \ldots, n)$. Let $m$ and $M$ be positive numbers, such that

$$
m \leq\left(\frac{a}{b}\right)^{q / p} \leq M, \text { and } m \leq \frac{x_{i}}{y_{i}^{q / p}} \leq M, \quad i=1,2, \ldots, n
$$

If $p>1$, then

$$
\frac{\left(a+c \sum_{i=1}^{n} x_{i}^{p}\right)^{\frac{1}{p}}}{b+c \sum_{i=1}^{n} x_{i} y_{i}} \leq \frac{1}{K(p, m, M)} \frac{\left(a+c \sum_{i=1}^{n} z_{i}^{p}\right)^{\frac{1}{p}}}{b+c \sum_{i=1}^{n} z_{i} y_{i}}
$$

If $p<1(p \neq 0)$, the reverse inequality holds in (14).

The next theorem give us a refinement of the above-mentioned converse of the Beckenbach inequality.

Theorem 5. Suppose the same conditions as in Theorem 4 hold. If $p>1$, then

$$
\begin{aligned}
\frac{\left(a+c \sum_{i=1}^{n} x_{i}^{p}\right)^{\frac{1}{p}}}{b+c \sum_{i=1}^{n} x_{i} y_{i}} & \leq \frac{1}{K(p, m, M)} \frac{\left(a+c \sum_{i=1}^{n} z_{i}^{p}\right)^{\frac{1}{p}}}{b+c \sum_{i=1}^{n} z_{i} y_{i}}\left(1-\frac{N(p, m, M) \Delta}{\left.b+c \sum_{i=1}^{n} x_{i} y_{i}\right)}\right. \\
& \leq \frac{1}{K(p, m, M)} \frac{\left(a+c \sum_{i=1}^{n} z_{i}^{p}\right)^{\frac{1}{p}}}{b+c \sum_{i=1}^{n} z_{i} y_{i}}
\end{aligned}
$$

where

$$
\Delta=\frac{M-m}{2}\left(a^{-\frac{q}{p}} b^{q}+c \sum_{i=1}^{n} y_{i}^{q}\right)-\left|b-\frac{m+M}{2 a^{q / p} b^{-q}}\right|-c \sum_{i=1}^{n}\left|x_{i} y_{i}-\frac{m+M}{2} y_{i}^{q}\right|
$$

and $K(p, m, M)$ and $N(p, m, M)$ are defined as in Theorem 3. If $p<1(p \neq 0)$, the reverse inequalities hold.

Proof. Let $p>1$. From equality $\frac{q}{p}+1=q$ we have

$$
\left(\frac{a y_{i}}{b}\right)^{\frac{q}{p}} y_{i}=\left(\frac{a}{b}\right)^{\frac{q}{p}} y_{i}^{q}
$$


and using that equality follows

$$
\frac{\left(a+c \sum_{i=1}^{n} z_{i}^{p}\right)^{\frac{1}{p}}}{b+c \sum_{i=1}^{n} z_{i} y_{i}}=\frac{\left(a+\left(\frac{a}{b}\right)^{q} c \sum_{i=1}^{n} y_{i}^{q}\right)^{\frac{1}{p}}}{b+\left(\frac{a}{b}\right)^{\frac{q}{p}} c \sum_{i=1}^{n} y_{i}^{q}}=\left(a^{-\frac{q}{p}} b^{q}+c \sum_{i=1}^{n} y_{i}^{q}\right)^{-\frac{1}{q}}
$$

Let us define a functional $A$. Let $\omega=\left(\omega_{1}, \ldots, \omega_{n+1}\right)$ be an $n$-tuple of non-negative real numbers. For a function $f:\{1,2, \ldots, n+1\} \rightarrow\langle 0, \infty\rangle, f(i)=f_{i}, A(f)$ is defined as

$$
A(f):=\sum_{i=1}^{n+1} \omega_{i} f_{i}
$$

Obviously, a functional $A$ is isotonic and linear.

For functions $f, g:\{1,2, \ldots, n+1\} \rightarrow\langle 0, \infty\rangle, f(i)=f_{i}, g(i)=g_{i}$, and the abovedefined functional $A$ inequality (6) becomes

$$
K(p, m, M)\left(\sum_{i=1}^{n+1} \omega_{i} f_{i}^{p}\right)^{\frac{1}{p}}\left(\sum_{i=1}^{n+1} \omega_{i} g_{i}^{q}\right)^{\frac{1}{q}}+\Delta\left(g^{q}, f g\right) N(p, m, M) \leq \sum_{i=1}^{n+1} \omega_{i} f_{i} g_{i}
$$

Using (16) with: $f_{1}=a^{\frac{1}{p}}, f_{2}=x_{1}, \ldots, f_{n+1}=x_{n}, g_{1}=a^{-\frac{1}{p}}, g_{2}=y_{1}, \ldots, g_{n+1}=$ $y_{n}, \omega_{1}=1, \omega_{2}=\cdots=\omega_{n+1}=c$, we obtain

$$
\begin{aligned}
\left(a+c \sum_{i=1}^{n} x_{i}^{p}\right)^{\frac{1}{p}} & \left(a^{-\frac{q}{p}} b^{q}+c \sum_{i=1}^{n} y_{i}^{q}\right)^{\frac{1}{q}} \\
\leq & \frac{1}{K(p, m, M)}\left(b+c \sum_{i=1}^{n} x_{i} y_{i}-\Delta \cdot N(p, m, M)\right)
\end{aligned}
$$

where $K$ and $N$ are defined as in Theorem 3 and $\Delta$ is defined as in Theorem 5 . Dividing the above inequality with $\left(b+c \sum_{i=1}^{n} x_{i} y_{i}\right)\left(a^{-q / p} b^{q}+c \sum_{i=1}^{n} y_{i}^{q}\right)^{1 / q}$ and using result (15) we obtain the desired refinement.

\section{The Converse Minkowski Inequality and Its Refinements}

In this section, we investigate the converse Minkowski inequality for functionals and the converse of the continuous form of the Minkowski inequality. In [9] (p. 116), the following converse Minkowski inequality for functionals is obtained.

Theorem 6 (The converse Minkowski inequality for functionals, [9]). Let $A, p, q, w, f, g$ satisfy assumptions of Theorem 3 with additional property $w(f+g)^{p} \in$ L. Let $m$ and $M$ be such that $0<m<f(x)(f(x)+g(x))^{-1} \leq M$ and $0<m<g(x)(f(x)+g(x))^{-1} \leq M$ for $x \in E$.

If $p>1$, then

$$
A^{\frac{1}{p}}\left(w(f+g)^{p}\right) \geq K(p, m, M) \cdot\left(A^{\frac{1}{p}}\left(w f^{p}\right)+A^{\frac{1}{p}}\left(w g^{p}\right)\right)
$$

where $K(p, m, M)$ is defined as in (2).

If $0<p<1$ or if $p<0$, then the reverse inequality in (17) holds provided that $A\left(w(f+g)^{p}\right)>0$ for $p<0$.

By using the refinement of the converse Hölder inequality we obtain the following improvement of the converse Minkowski inequality for functionals. 
Theorem 7. Suppose the same conditions as in Theorem 6 hold. If $p>1$, then

$$
\begin{gathered}
A^{\frac{1}{p}}\left(w(f+g)^{p}\right) \geq K(p, m, M)\left(A^{\frac{1}{p}}\left(w f^{p}\right)+A^{\frac{1}{p}}\left(w g^{p}\right)\right) \\
+\quad N(p, m, M) \frac{\Delta\left((f+g)^{p}, f(f+g)^{p-1}\right)+\Delta\left((f+g)^{p}, g(f+g)^{p-1}\right)}{A^{1-\frac{1}{p}}\left(w(f+g)^{p}\right)} .
\end{gathered}
$$

If $p<1(p \neq 0)$, then the reversed inequality holds.

Proof. Let $p>1$. Writing $A\left(w(f+g)^{p}\right)$ as

$$
A\left(w(f+g)(f+g)^{p-1}\right)=A\left(w f(f+g)^{p-1}+w g(f+g)^{p-1}\right),
$$

and using inequality (6) we obtain

$$
\begin{aligned}
& A\left(w(f+g)^{p}\right)=A\left(w f(f+g)^{p-1}\right)+A\left(w g(f+g)^{p-1}\right) \\
\geq & K(p, m, M) A^{\frac{1}{p}}\left(w f^{p}\right) A^{\frac{1}{q}}\left(w(f+g)^{p}\right) \\
+ & \Delta\left((f+g)^{p}, f(f+g)^{p-1}\right) N(p, m, M) \\
+ & K(p, m, M) A^{\frac{1}{p}}\left(w g^{p}\right) A^{\frac{1}{q}}\left(w(f+g)^{p}\right) \\
+ & \Delta\left((f+g)^{p}, g(f+g)^{p-1}\right) N(p, m, M) \\
= & K(p, m, M) A^{\frac{1}{q}}\left(w(f+g)^{p}\right)\left(A^{\frac{1}{p}}\left(w f^{p}\right)+A^{\frac{1}{p}}\left(w g^{p}\right)\right) \\
+ & N(p, m, M)\left(\Delta\left((f+g)^{p}, f(f+g)^{p-1}\right)+\Delta\left((f+g)^{p}, g(f+g)^{p-1}\right)\right) .
\end{aligned}
$$

Dividing by $A^{\frac{1}{q}}\left(w(f+g)^{p}\right)$ we obtain desired result.

If $p>1$, then the second term in the sum on the right-hand side in (18) is non-negative and inequality (18) is a refinement of the known converse (17). Similar proof holds for $p<1,(p \neq 0)$.

The previous investigation does not cover the so-called Minkowski integral inequality. Namely, if $\left(X, \Sigma_{X}, \mu\right)$ and $\left(Y, \Sigma_{Y}, v\right)$ are two measure spaces with sigma-finite measures $\mu$ and $v$, respectively, and if $f$ is a non-negative function on $X \times Y$ which is integrable with respect to the measure $\mu \times v$, then for $p \geq 1$ we obtain

$$
\left[\int_{X}\left(\int_{Y} f(x, y) d \nu(y)\right)^{p} d \mu(x)\right]^{\frac{1}{p}} \leq \int_{Y}\left(\int_{X} f^{p}(x, y) d \mu(x)\right)^{\frac{1}{p}} d \nu(y) .
$$

The above result is also called "the continuous form of the Minkowski inequality" or "the Minkowski inequality for infinitely many functions" and, for example, it can be found in [12] (p. 41). Considering the proof of this inequality we can conclude that there exist a related result for other values of the exponent $p$ (see [13]).

If $0<p<1$ and

$$
\int_{X}\left(\int_{Y} f(x, y) d \nu(y)\right)^{p} d \mu(x)>0, \int_{Y} f(x, y) d \nu(y)>0,(\mu-\text { a.e. })
$$

then the reverse inequality holds.

If $p<0$, the above-mentioned assumptions (20) and the additional one

$$
\int_{X} f^{p}(x, y) d \mu(x)>0 \quad(v-\text { a.e. })
$$

then the reverse inequality holds. 
To our knowledge, in the literature there is no result corresponding to the conversing of the above mentioned results. In the next theorem, we state a converse and a refinement of that variant of the Minkowski inequality. The proof is based on the proof of the continuous form of the Minkowski inequality and on the use of result of Theorem 3.

Theorem 8 (The converse continuous form of the Minkowski inequality and refinement). Let $\left(X, \Sigma_{X}, \mu\right)$ and $\left(Y, \Sigma_{Y}, v\right)$ be two measure spaces with sigma-finite measures $\mu$ and $v$ respectively. Let $f$ be a non-negative function on $X \times Y$, integrable with respect to the measure $(\mu \times v)$.

$$
\begin{gathered}
\text { If } 0<m \leq \frac{f(x, y)}{\int_{Y} f(x, y) d v(y)} \leq M \text { for all } y \in Y, x \in X, \text { then for } p \geq 1 \\
\quad\left[\int_{X}\left(\int_{Y} f(x, y) d v(y)\right)^{p} d \mu(x)\right]^{\frac{1}{p}} \\
\geq K(p, m, M) \int_{Y}\left(\int_{X} f^{p}(x, y) d \mu(x)\right)^{\frac{1}{p}} d v(y) \\
+N(p, m, M)\left[\int_{X} H^{p}(x) d \mu(x)\right]^{\frac{1-p}{p}} \Delta_{1} \\
\geq K(p, m, M) \int_{Y}\left(\int_{X} f^{p}(x, y) d \mu(x)\right)^{\frac{1}{p}} d v(y),
\end{gathered}
$$

where $K(p, m, M)$ is defined with (2), $N(p, m, M)$ is defined with (12), $H(x)=\int_{Y} f(x, y) d v(y)$ and

$$
\Delta_{1}=\int_{Y}\left(\int_{X}\left(\frac{m-M}{2} H^{p}(x)-\left|f(x, y) H^{p-1}(x)-\frac{m+M}{2} H^{p}(x)\right|\right) d \mu(x)\right) d v(y) .
$$

If $0<p<1$ with (20) or $p<0$ with (20) and (21), then the reversed inequality holds.

Proof. Let us denote

$$
H(x)=\int_{Y} f(x, y) d v(y)
$$

Using Fubini's theorem we obtain

$$
\begin{gathered}
\int_{X}\left(\int_{Y} f(x, y) d v(y)\right)^{p} d \mu(x)=\int_{X} H^{p}(x) d \mu(x) \\
=\int_{X}\left(\int_{Y} f(x, y) d v(y)\right) H^{p-1}(x) d \mu(x)=\int_{Y}\left(\int_{X} f(x, y) H^{p-1}(x) d \mu(x)\right) d v(y) .
\end{gathered}
$$

Using (7) with functional $A(\phi)=\int_{X} \phi(x) d \mu(x)$ we obtain

$$
\begin{gathered}
\int_{Y}\left(\int_{X} f(x, y) H^{p-1}(x) d \mu(x)\right) d v(y) \\
\geq K(p, m, M) \int_{Y}\left(\int_{X} f^{p}(x, y) d \mu(x)\right)^{1 / p}\left(\int_{X} H^{p}(x) d \mu(x)\right)^{\frac{p-1}{p}} d v(y)+N(p, m, M) \Delta_{1} \\
\geq K(p, m, M) \int_{Y}\left(\int_{X} f^{p}(x, y) d \mu(x)\right)^{1 / p}\left(\int_{X} H^{p}(x) d \mu(x)\right)^{\frac{p-1}{p}} d v(y) .
\end{gathered}
$$

Dividing by $\left(\int_{X} H^{p}(x) d \mu(x)\right)^{\frac{p-1}{p}}$ we obtain inequalities (22) and (23). 


\section{Applications on Mixed Means}

It is interesting to show how the previously obtained results impact to the study of mixed means.

Let $r, s$ be two positive numbers, $r<s$. Replacing $p$ by $\frac{s}{r}$, replacing $f$ by $f^{r}$ in inequalities (19) and (23), raising to the power $\frac{1}{r}$ and dividing with $(\mu(X))^{1 / s}$ and $(\nu(Y))^{1 / r}$, we obtain

$$
\left[\frac{\int_{X}\left(\frac{\int_{Y} f^{r}(x, y) d v(y)}{v(Y)}\right)^{\frac{s}{r}} d \mu(x)}{\mu(X)}\right]^{\frac{1}{s}} \leq\left[\frac{\int_{Y}\left(\frac{\int_{X} f^{s}(x, y) d \mu(x)}{\mu(X)}\right)^{\frac{r}{s}} d v(y)}{v(Y)}\right]^{\frac{1}{r}},
$$

and

$$
\begin{gathered}
{\left[\frac{\int_{X}\left(\frac{\int_{Y} f^{r}(x, y) d v(y)}{v(Y)}\right)^{\frac{s}{r}} d \mu(x)}{\mu(X)}\right]^{\frac{1}{s}}} \\
\geq K^{\frac{1}{r}}\left(\frac{s}{r}, m, M\right)\left[\frac{\int_{Y}\left(\frac{\int_{X} f^{S}(x, y) d \mu(x)}{\mu(X)}\right)^{\frac{r}{s}} d v(y)}{v(Y)}\right]^{\frac{1}{r}},
\end{gathered}
$$

where $m$ and $M$ are real numbers, such that $0<m \leq \frac{f^{r}(x, y)}{\int_{Y} f^{r}(x, y) d v(y)} \leq M$.

Using notation

$$
M^{[r]}(f, \mu)= \begin{cases}\left(\frac{\int_{X} f^{r}(x) d \mu(x)}{\mu(X)}\right)^{\frac{1}{r}} ; & r \neq 0 \\ \exp \left(\frac{\int_{X} \log f(x) d \mu(x)}{\mu(X)}\right) ; & r=0\end{cases}
$$

in inequalities (24) and (25) we obtain the following theorem for mixed means.

Theorem 9. Suppose the same conditions as in Theorem 8 hold. If $r<s, r, s \neq 0$, then

$$
M^{[s]}\left(M^{[r]}(f, v), \mu\right) \leq M^{[r]}\left(M^{[s]}(f, \mu), v\right) .
$$

If $m$ and $M$ are real numbers, such that $0<m \leq \frac{f^{r}(x, y)}{\int_{Y} f^{r}(x, y) d v(y)} \leq M$, then

$$
M^{[s]}\left(M^{[r]}(f, v), \mu\right) \geq K^{\frac{1}{r}}\left(\frac{s}{r}, m, M\right) \cdot M^{[r]}\left(M^{[s]}(f, \mu), v\right)
$$

where $K$ is defined by (2).

Additionally, by using (22) the refinement of the above mixed mean inequality can be obtained.

These inequalities are inequalities for mixed means, the second one is a converse of the first inequality. Discrete version of (26) is given in [10] (p. 109), while its conversion is a new result. It is instructive to calculate mixed means for some special spaces and measures.

Corollary 1. Let $a, b, \alpha, \gamma, r, s \in \mathbb{R}$ be such that $a<b, r<s, \alpha, \gamma>0, r, s \neq 0$. If $g:[a, b] \rightarrow \mathbb{R}$ is a non-negative measurable function, then the following inequality holds:

$$
\left[\frac{1}{(b-a)^{\gamma}} \int_{a}^{b}(y-a)^{\gamma-1}\left(\frac{1}{(y-a)^{\alpha}} \int_{a}^{y} g^{r}(t)(t-a)^{\alpha-1} d t\right)^{\frac{s}{r}} d y\right]^{\frac{1}{s}}
$$




$$
\leq\left[\frac{1}{(b-a)^{\alpha}} \int_{a}^{b}(y-a)^{\alpha-1}\left(\frac{1}{(y-a)^{\gamma}} \int_{a}^{y} g^{s}(t)(t-a)^{\gamma-1} d t\right)^{\frac{r}{s}} d y\right]^{\frac{1}{r}} .
$$

Furthermore, if $m$ and $M$ are real numbers, such that for $x \in[0,1], y \in[a, b]$

$$
0<m \leq \frac{g^{r}(a+x(y-a))}{\int_{a}^{b} g^{r}(a+x(y-a))(y-a)^{\alpha-1} d y} \leq M,
$$

then

$$
\begin{gathered}
{\left[\frac{1}{(b-a)^{\gamma}} \int_{a}^{b}(y-a)^{\gamma-1}\left(\frac{1}{(y-a)^{\alpha}} \int_{a}^{y} g^{r}(t)(t-a)^{\alpha-1} d t\right)^{\frac{s}{r}} d y\right]^{\frac{1}{s}}} \\
\geq K^{\frac{1}{r}}\left(\frac{s}{r}, m, M\right) \cdot\left[\frac{1}{(b-a)^{\alpha}} \int_{a}^{b}(y-a)^{\alpha-1}\left(\frac{1}{(y-a)^{\gamma}} \int_{a}^{y} g^{S}(t)(t-a)^{\gamma-1} d t\right)^{\frac{r}{s}} d y\right]^{\frac{1}{r}} .
\end{gathered}
$$

Proof. Using (26) with the following: $X=[0,1], Y=[a, b], d \mu(x)=x^{\gamma-1} d x$ and $d v(y)=(y-a)^{\alpha-1} d y, \alpha, \gamma \in \mathbb{R} \backslash\{0\}, f(x, y)=g(a+x(y-a))$ where $g$ is a non-negative measurable function. Then, $v(Y)=\frac{1}{\alpha}(b-a)^{\alpha}$ and $\mu(X)=\frac{1}{\gamma}$.

After substitutions it follows

$$
\begin{aligned}
& {\left[\frac{\gamma \alpha^{s / r}}{(b-a)^{\alpha / r}} \int_{0}^{1}\left(\int_{a}^{b} g^{r}(a+x(y-a))(y-a)^{\alpha-1} d y\right)^{\frac{s}{r}} x^{\gamma-1} d x\right]^{\frac{1}{s}}} \\
& \leq\left[\frac{\gamma^{r / s} \alpha}{(b-a)^{\alpha}} \int_{a}^{b}\left(\int_{0}^{1} g^{s}(a+x(y-a)) x^{\gamma-1} d x\right)^{\frac{r}{s}}(y-a)^{\alpha-1} d y\right]^{\frac{1}{r}} .
\end{aligned}
$$

Replacing in the right-hand side of inequality $a+x(y-a)$ with the new variable $t$, we obtain

$$
\left[\frac{\gamma^{r / s} \alpha}{(b-a)^{\alpha}} \int_{a}^{b}\left(\frac{1}{(y-a)^{\gamma}} \int_{a}^{y} g^{s}(t)(t-a)^{\gamma-1} d t\right)^{\frac{r}{s}}(y-a)^{\alpha-1} d y\right]^{\frac{1}{r}} .
$$

The same substitution is done in the left-hand side of (30) and we obtain that the left-hand side is equal to:

$$
\left[\frac{\gamma \alpha^{s / r}}{(b-a)^{\alpha s / r}} \int_{0}^{1} x^{\gamma-1}\left(\frac{1}{x^{\alpha}} \int_{a}^{a+x(b-a)} g^{r}(t)(t-a)^{\alpha-1} d t\right)^{\frac{s}{r}} d x\right]^{\frac{1}{s}} .
$$

Replacing $a+x(b-a)$ with the new variable $y$ in the outer integral, it is equal to

$$
\left[\frac{\gamma \alpha^{s / r}}{(b-a)^{\alpha s / r}} \int_{a}^{b} \frac{(y-a)^{\gamma-1}}{(b-a)^{\gamma-1}}\left(\frac{(b-a)^{\alpha}}{(y-a)^{\alpha}} \int_{a}^{y} g^{r}(t)(t-a)^{\alpha-1} d t\right)^{\frac{s}{r}} \frac{d y}{b-a}\right]^{\frac{1}{s}} .
$$

Finally, we obtain

$$
\begin{aligned}
& {\left[\frac{1}{(b-a)^{\gamma}} \int_{a}^{b}(y-a)^{\gamma-1}\left(\frac{1}{(y-a)^{\alpha}} \int_{a}^{y} g^{r}(t)(t-a)^{\alpha-1} d t\right)^{\frac{s}{r}} d y\right]^{\frac{1}{s}} } \\
\leq & {\left[\frac{1}{(b-a)^{\alpha}} \int_{a}^{b}(y-a)^{\alpha-1}\left(\frac{1}{(y-a)^{\gamma}} \int_{a}^{y} g^{s}(t)(t-a)^{\gamma-1} d t\right)^{\frac{r}{s}} d y\right]^{\frac{1}{r}}, }
\end{aligned}
$$

where $\alpha, \gamma>0, r<s, r, s \neq 0$. From (25) with same substitutions we obtain a converse of (28). 
Let us point out that inequality (28) was firstly obtained in [14] (Theorem 3) and it was used for proving the well-known Hardy inequality. Additionally, let us mention that the above inequalities about mixed means can be refined like as inequalities in previous sections.

\section{Conclusions}

In this paper, we have obtained the refinement of the converse Hölder inequality which follows from a monotonicity of the discrete Jensen functional. Additionally, a new conversion of the continuous form of the Minkowski inequality is proved. Our main method in the present work is to use the recently obtained refinement of the converse Hölder inequality in finding refinements of converses of the Beckenbach and the Minkowski inequalities. It would be interesting to explore whether our method can be used to find refinements of other inequalities. Thus, the study of the refinement of other inequalities is a suggested future work.

Author Contributions: Writing-original draft, J.P. (Josip Pečarić), J.P. (Jurica Perić) and S.V. All authors have read and agreed to the published version of the manuscript.

Funding: The research of first author was supported by the RUDN University Strategic Academic Leadership Program.

Institutional Review Board Statement: Not applicable.

Informed Consent Statement: Not applicable.

Data Availability Statement: Not applicable.

Conflicts of Interest: The authors declare no conflict of interest.

\section{References}

1. Barić, J.; Bibi, R.; Bohner, M.; Nosheen, A.; Pečarić, J. Jensen Inequalities on Time Scales; Element: Zagreb, Croatia, 2015.

2. Debnath, P.; de La Sen, M. Set-valued Interpolative Hardy-Rogers and Set-valued Reich-Rus-Ćirić-type Contractions in $b$-Metric Spaces. Mathematics 2019, 7, 849. [CrossRef]

3. Debnath, P.; de La Sen, M. Contractive Inequalities for Some Asymptotically Regular Set-Valued Mappings and Their Fixed Points. Symmetry 2020, 12, 411. [CrossRef]

4. Matković, A.; Perić, J. Refinement of the Jessen-Mercer inequality and a generalization on convex hulls in $\mathbf{R}^{k}$. J. Math. Inequal. 2015, 9, 1093-1114. [CrossRef]

5. Mićić Hot, J.; Perić, J.; Pečarić, J. Refined Jensen's operator inequality with condition on spectra. Oper. Matrices 2013, 7, $293-308$. [CrossRef]

6. $\quad$ Furuta, T.; Hot, J.M.; Pečarić, J.; Seo, Y. Mond-Pečarić Method in Operator Inequalities; Element: Zagreb, Croatia, 2005.

7. Mond, B.; Pečarić, J.; Šunde, J.; Varošanec, S. Operator versions of some classical inequalities. Linear Algebra Its Appl. 1997, 264, 117-126. [CrossRef]

8. Nikolova, L.; Persson, L.-E.; Varošanec, S. A new look at classical inequalities involving Banach lattice norms. J. Inequal. Appl. 2017, 2017, 302. [CrossRef] [PubMed]

9. Pečarić, J.E.; Proschan, F.; Tong, Y.L. Convex Functions, Partial Orderings, and Statistical Applications; Academic Press Inc.: Boston, MA, USA, 1992.

10. Mitrinović, D.S.; Pečarić, J.E.; Fink, A.M. Classical and New Inequalities in Analysis; Kluwer Acad. Publishers: Dordrecht, The Netherlands, 1993.

11. Zhuang, Y.-D. The Beckenbach inequality and its inverse. J. Math. Anal. Appl. 1993, 175, 118-125. [CrossRef]

12. Lieb, E.H.; Loss, M. Analysis. In Graduate Studies in Mathematics; American Mathematical Society: Providence, RI, USA, 2001; Volume 14

13. Ivanković, B.; Pečarić, J.; Varošanec, S. Properties of mappings related to the Minkowski inequality. Mediterr. J. Math. 2011, 8, 543-551. [CrossRef]

14. Čižmešija, A.; Pečarić, J. Mixed means and Hardy's inequality. Math. Inequal. Appl. 1998, 1, 491-506. [CrossRef] 